\title{
The Calculation of the Perturbative Expansion of Wilson Operators on Lattice
}

\author{
Da Qing $\mathrm{Liu}^{2}$ and Ji Min $\mathrm{Wu}^{1,2}$ \\ ${ }^{1}$ CCAST(World Lab. ), P. O. Pox 8730, Beijing 100080, China \\ ${ }^{2}$ Institute of High Energy Physics, Chinese Academy of Sciences, \\ P. O. Box 918-4, Beijing, 100039, P. R. China
}

November 21, 2018

\begin{abstract}
We introduce an approach to expand gauge-invariant Wilson operators on lattice. This approach is based on non-abelian Stokes theorem and overcomes some shortage of some former methods. It is also suitable for expanding any Wilson operator on lattice.
\end{abstract}




\section{Introduction}

Expanding gauge-invariant Wilson operators, or, Wilson loops, according to lattice spacing $a$ is a very important work when we do theoretical analysis in lattice QCD. It is a necessary step in many cases, such as the discussion of improved action 11 , the exploration of improved cooling 2 or the investigate for renormalization procedure 3 when study instanton phenomenon, etc. Furthermore, in order to calculate masses of glueball states, we developed a new opinion[ 4 , in which the perturbative expansion of Wilson loops is also a needed progress.

Due to the complicated of path ordering operators, it is cockamamie and difficult to expand the Wilson loops according to normal method if the number of links of Wilson loop is large or it is needed to expand Wilson loop up to higher precision. There is a prevalent approach introduced by Luscher and Weisz in ref.[0]. But this approach has some shortcoming, for instance, one is likely to face some difficult when he tries to expand relatively complicated spatial loops.

We introduce a practical easy approach which is based on the non-abelian Stokes theorem here. This approach has overcome the shortcoming in ref. [5] and is therefore suitable for any Wilson loops on lattice. It is also suitable for any of above cases in ref. [1, 2, 3, 田. To do this, we will give a short comment on Luscher-Weisz method in the following section and some mathematical preparation in section 3 . We exemplify our method in section 4.

Since the case in ref. [1] is the most difficult and universal one, we should pay our most attention on this case.

\section{The Comment on Luscher-Weisz Method}

Let us observe an arbitrary gauge-invariant Wilson loop on the cubic lattice. In order to get the perturbative expansion of the loop up to $O\left(a^{6}\right)$ ( To simplify, this paper only discusses the expansion up to $\left.O\left(a^{6}\right)\right)$, Luscher and Weisz adopt the following method: they fixed a gauge at first, then try to rewrite it in gaugeinvariant form.

Assume that one needs to expand the plaquette operator:

$$
p l=\sum_{n} \sum_{\mu \nu} p l_{\mu \nu}(n)=\sum_{n} \sum_{\mu, \nu} \operatorname{Tr}\left\{U(n, \mu) U(n+\widehat{\mu}, \nu) U^{-1}(n+\widehat{\nu}, \mu) U^{-1}(n, \nu)\right\},
$$

where $\widehat{\mu}$ is the unit vector along $\mu$ direction, $U(n, \mu)$ is link variable defined as follows:

$$
U(n, \mu)=T \exp a \int_{0}^{1} d t A_{\mu}(a n+a \widehat{\mu} t),
$$

in which $A_{\mu}$ is gauge potential' We point out here that the summation is different following different problem. For instance, the summation of $n$ is over all the 4-

\footnotetext{
${ }^{1}$ We use a notation here that $A_{\mu}$ is anti-hermitian, traceless $N \times N$-matrix
} 
dimensional lattice sites and the summation of directions $(\mu \nu)$ is over all possible 4dimensional space-time directions when one discusses the improved action 11 , while the summation of $n$ is over spatial lattice sites in the same time slice and that of directions is variant in 3-dimensional spatial directions according to different problems in the case of ref. [4].

One can always choose a proper local gauge on lattice to make the gauge field satisfy

$$
\begin{aligned}
& A_{\mu}(x)=0, \quad \text { for all } x, \\
& A_{\nu}(x)=0, \quad \text { for all } x \text { with } x_{\mu}=0 .
\end{aligned}
$$

$p l_{\mu \nu}(0)$ then reduces to

$$
p l_{\mu \nu}(0)=\operatorname{Tr} T \exp a \int_{0}^{1} d t A_{\mu}(a \widehat{\mu}+a \widehat{\mu} t) .
$$

At the same time, at $x=0$, one has

$$
\partial_{\nu}^{p} \partial_{\mu}^{q+1} A_{\nu}=D_{\nu}^{p} D_{\mu}^{q} F_{\mu \nu}
$$

Insert Eq. (4) into Eq. (3) and expand Eq. (3) according to lattice spacing $a$ :

$$
\begin{aligned}
p l_{\mu \nu}(0) & =N+\frac{1}{2} a^{4} \operatorname{Tr}\left(F_{\mu \nu} F_{\mu \nu}\right)+\frac{1}{2} a^{5} \operatorname{Tr}\left[F_{\mu \nu}\left(D_{\mu}+D_{\nu}\right) F_{\mu \nu}\right] \\
& +\frac{1}{6} a^{6} \operatorname{Tr}\left\{F_{\mu \nu}^{3}+F_{\mu \nu}\left(D_{\mu}^{2}+\frac{3}{2} D_{\mu} D_{\nu}+D_{\nu}^{2}\right) F_{\mu \nu}\right. \\
& \left.+\frac{3}{4}\left(D_{\mu}+D_{\nu}\right) F_{\mu \nu}\left(D_{\mu}+D_{\nu}\right) F_{\mu \nu}\right\}+O\left(a^{7}\right),
\end{aligned}
$$

where $F_{\mu \nu}=\partial_{\mu} A_{\nu}-\partial_{\nu} A_{\mu}+\left[A_{\mu}, A_{\nu}\right]$ and $D_{\mu} F_{\nu \rho}=\partial_{\mu} F_{\nu \rho}+\left[A_{\mu}, F_{\nu \rho}\right]$. Due to periodic boundary condition, one can ignore all the (covariant) differential terms in the last result( Section 4 shows more details) and gets:

$$
p l_{\mu \nu}(0)=\frac{1}{2} a^{4} \operatorname{Tr} F_{\mu \nu}^{2}-\frac{1}{24} a^{6} \operatorname{Tr}\left[F_{\mu \nu}\left(D_{\mu}^{2}+D_{\nu}^{2}\right) F_{\mu \nu}\right]+\frac{1}{6} a^{6} \operatorname{Tr} F_{\mu \nu}^{3}+O\left(a^{7}\right) .
$$

Now let us observe Eq. (雨). In this case, because

$$
D_{\mu} D_{\nu}-D_{\nu} D_{\mu}=\left[F_{\mu \nu} \cdot \cdot\right]
$$

the corresponding in Eq. (4) is unambiguous. But, when one tries to expand some complicated spatial Wilson operators, l.h.s. of Eq. (4) is possible as the form $\partial_{\mu} \partial_{\nu} A_{\rho}(\mu \neq \nu \neq \rho)$. There is ambiguity that such terms may correspond both to $D_{\mu} F_{\nu \rho}$ and to $D_{\nu} F_{\mu \rho}$, which means one can not determine the expansion unambiguously, especially when the summation of directions is not over all possible directions but some definite ones 4 . 
In the case of ref. [1, 5] that one should sum over all possible 4-dimensional directions, Luscher and Weisz eliminate the ambiguity through the presumption that the expansion of any Wilson loop after summation over all possible directions and lattice sites is invariant under any Lorentz transformation( In 4-dimensional Euclidean space, it is $S O(4)$ group), parity and charge conjugacy transformation. This presumption will some time complicate our expansion, furthermore, it has not been proved strictly. We show a debate here. Suppose we accepts this presumption and restricts ourselves into 3-dimensional space, the subspace of the 4-dimensional Euclidean space and time. One natural deduction is that the operator, which has been summed over all space lattice sites in the same time slice and all possible 3dimensional directions, is invariant under $S O(3)^{P C}$ group since the $S O(3)$ group is the subgroup of the $S O(4)$ group. As such operator belongs to representation $A_{1}^{++}$, the trivial representation of the cubic group on lattice, one may regard that the $J^{P C}$ ( in continuum limit) of the operator belonging to $A_{1}^{++}$is $0^{++}$. In other words, the subduced representation of the representation $0^{++}$of the $S O(3)^{P C}$ group can be reduced into $A_{1}^{++}$( subduced representation: the representation which is obtained by trivially embedding the cubic group $O^{P C}$ into the group $\left.S O(3)^{P C}\right)$. But, as we know, there also exist other representations in the group $S O(3)^{P C}$, the subduced representation of which can be reduced into $A_{1}^{++}$, for instance, $4^{++}$6, 7], which means that the $J^{P C}$ ( in the continuum limit) of the operator belonging to $A_{1}^{++}$ is not always $0^{++}$. So, the validity of the presumption is doubtful. In fact, a discussion in ref. [10 does not support this presumption too. To say the least, even this presumption is right, one can hardly apply it to expand the operator, for instance, the $P C$ of which is not ++ .

To solve the problem, we developed a new approach which can determine the perturbative expansion unambiguously. The next section is the proper mathematical preparation.

\section{Mathematical Preliminary}

For operators, or loops, the traces over color space are not performed in this section, so, these operators are gauge covariant under gauge transformation.

Now we begin our preparation with some simple examples.

Observe the operator $\tilde{W}(O, Z)$ shown in Fig. 1:

$$
\tilde{W}(O, Z)=U_{R}\left(Z, O ; C_{X}\right) W(Z) U_{R}\left(O, Z ; \bar{C}_{X}\right),
$$

where $W$ is a definite gauge covariant operator which is from point $Z$ and back to $Z$, and $U_{R}\left(Z, O, C_{X}\right)=P \exp \int_{0}^{x} d t A_{i}(\hat{i t})$ is connector. Some properties of connectors are list as follows [8]:

a)

$$
U_{R}\left(X_{3}, X_{1} ; C_{1}+C_{2}\right)=U_{R}\left(X_{2}, X_{1} ; C_{1}\right) \cdot U_{R}\left(X_{3}, X_{2} ; C_{2}\right)
$$

where $C_{1}$ is a curve going from point $X_{1}$ to point $X_{2}$ and $C_{2}$ is a curve going from point $X_{2}$ to point $X_{3}$. 
b)

$$
\begin{array}{r}
U_{R}(X, Y ; \bar{C}) U_{R}(Y, X ; C)=1, \\
U_{R}^{\dagger}(Y, X, C)=U_{R}(X, Y ; \bar{C}),
\end{array}
$$

$C$ is a curve from $X$ to $Y$ and $\bar{C}$ the same curve but oriented in inverse direction, running from $Y$ to $X$.

From the opinion of differential geometry, $\tilde{W}$ is the shifted version of operator $W$ from point $Z$ to point $O$.

Under local gauge transform $V$ we have:

$$
\begin{aligned}
\tilde{W}(O, Z) & \rightarrow V(O) \tilde{W}(O, Z) V^{-1}(O) \\
W(Z) & \rightarrow V(Z) W(Z) V^{-1}(Z) .
\end{aligned}
$$

Let $W(Z)=W+x \partial_{i} W+\cdots+\frac{x^{n}}{n !} \partial_{i}^{n} W+\cdots$, where $W \equiv W(O)$ is the same operator as $W(Z)$ but it is from $O$ and back to $O$.

We can prove that

$$
\tilde{W}(O, Z)=W+x D_{i} W+\cdots+\frac{x^{n}}{n !} D_{i}^{n} W+\cdots .
$$

Then we study the operator described in Fig. 2 ( We also denote it by $\tilde{W}$ ):

$$
\tilde{W}(O, Z)=U_{R}\left(Z, O ; C_{Y}+C_{X}\right) W(Z) U_{R}\left(O, Z ; \bar{C}_{X}+\bar{C}_{Y}\right) .
$$

We let $W(Z)=W+x \partial_{i} W+y \partial_{i} W+\frac{1}{2}\left(x^{2} \partial_{i}^{2}+2 x y \partial_{i} \partial_{j} W+y^{2} \partial_{j}^{2} W\right)+\cdots$, in which $W \equiv W(O)$. Using Eq. (12) and the properties of connector we get

$$
\tilde{W}(O, Z)=W+x D_{i} W+y D_{j} W+\frac{1}{2}\left(x^{2} D_{i}^{2}+2 x y D_{j} D_{i}+y^{2} D_{j}^{2}\right) W+\cdots .
$$

It should be pointed out here that, in general, the order of $D_{j} D_{i}$ can not be reversed in above equation.

Other more complicated case can be calculated as similar as above.

We write out non-abelian Stokes theorem as( We let $\left.t_{0} \equiv a\right)$ [9]

$$
\begin{aligned}
U_{\mu \nu}(x) & =U_{\mu}(x) U_{\nu}(x+\widehat{\mu}) U_{\mu}^{\dagger}(x+\widehat{\nu}) U_{\nu}^{\dagger}(x) \\
& =P \exp \int_{0}^{a} d s \int_{0}^{a} d t \tilde{F}_{\mu \nu}(x+s \widehat{\mu}+\widehat{\nu}) \\
& =1+\sum_{m=1}^{\infty} \prod_{i=1}^{m} \int_{0}^{a} d s_{i} \int_{0}^{t_{i-1}} d t_{i} \tilde{F}_{\mu \nu}\left(x+s_{n} \widehat{\mu}+t_{n} \widehat{\nu}\right) \cdots \tilde{F}_{\mu \nu}\left(x+s_{1} \widehat{\mu}+t_{1} \widehat{\mu}\right) .
\end{aligned}
$$

Here $\tilde{F}_{\mu \nu}(x+s \widehat{\mu}+t \widehat{\nu})$ is the shifted version of $F_{\mu \nu}(x+s \widehat{\mu}+t \widehat{\nu})$ from point $x+s \widehat{\mu}+t \widehat{\nu}$ to point $x$, or:

$$
V(s, t)=P \exp \int_{0}^{t} d t^{\prime} A_{\nu}\left(x+t^{\prime} \widehat{\nu}\right) \cdot P \exp \int_{0}^{s} d s^{\prime} A_{\mu}\left(x+t \widehat{\nu}+s^{\prime} \widehat{\mu}\right),
$$




$$
\tilde{F}_{\mu \nu}\left(x+s_{n} \widehat{\mu}+t_{n} \widehat{\nu}\right)=V(s, t) F_{\mu \nu}(x+s \widehat{\mu}+t \widehat{\nu}) V^{\dagger}(s, t) .
$$

Now using Eq. (14), (15), (16) we can get the expansion of the operator from $O$ back to $O$ which is shown in fig. 3 (a):

$$
\begin{aligned}
U_{i j}^{(1)}(n)= & U_{i}(n) U_{j}(n+\widehat{i}) U_{i}^{\dagger}(n+\widehat{j}) U_{j}^{\dagger}(n) \\
= & 1+a^{2} F_{i j}+\frac{a^{3}}{2}\left(D_{i}+D_{j}\right) F_{i j}+\frac{a^{4}}{6}\left(D_{i}^{2}+D_{j}^{2}+\frac{3}{2} D_{j} D_{i}\right) F_{i j} \\
& +P_{i j}+\frac{a^{6}}{6} F_{i j}^{3}+O\left(a^{7}\right)
\end{aligned}
$$

where $P_{i j}=\frac{a^{4}}{2} F_{i j}^{2}+\frac{a^{6}}{24} F_{i j}\left(D_{i}^{2}+D_{j}^{2}\right) F_{i j}, F_{i j}=F_{i j}(n)$. Because our precision in last result is $O\left(a^{6}\right)$, we have ignored all the full (covariant) differential terms the ranks of which are large than or equal to $O\left(a^{5}\right)$ ( More details are shown in section 4), for instance the term $\frac{a^{5}}{2} F_{i j}\left(D_{i}+D_{j}\right) F_{i j}$.

Using Eq. (17) and the properties of connector we get the perturbative expansion of the operator in Fig. 3(b):

$$
\begin{aligned}
U_{i j}^{(2)}(n)= & U_{j}(n) U_{i}^{\dagger}(n-\widehat{i}+\widehat{j}) U_{j}^{\dagger}(n-\widehat{i}) U_{i}(n-\hat{i}) \\
= & 1+a^{2} F_{i j}-\frac{a^{3}}{2}\left(D_{i}-D_{j}\right) F_{i j}+\frac{a^{4}}{6}\left(D_{i}^{2}+D_{j}^{2}-\frac{3}{2} D_{j} D_{i}\right) F_{i j} \\
& +P_{i j}+\frac{a^{6}}{6} F_{i j}^{3}+O\left(a^{7}\right) .
\end{aligned}
$$

With the same reason we get the perturbative expansion of the operator in Fig. $3(\mathrm{c})$ :

$$
\begin{aligned}
U_{i j}^{(3)}(n)= & U_{i}^{\dagger}(n-\hat{i}) U_{j}^{\dagger}(n-\widehat{i}-\hat{j}) U_{i}(n-\widehat{i}-\widehat{j}) U_{j}(n-\hat{j}) \\
= & 1+a^{2} F_{i j}-\frac{a^{3}}{2}\left(D_{i}+D_{j}\right) F_{i j}+\frac{a^{4}}{6}\left(D_{i}^{2}+D_{j}^{2}+\frac{3}{2} D_{j} D_{i}\right) F_{i j} \\
& +P_{i j}+\frac{a^{6}}{6} F_{i j}^{3}+O\left(a^{7}\right) .
\end{aligned}
$$

And the expansion of the operator in Fig. 3(d):

$$
\begin{aligned}
U_{i j}^{(3)}(n)= & U_{j}^{\dagger}(n-\hat{j}) U_{i}(n-\widehat{j}) U_{j}(n+\widehat{i}-\widehat{j}) U_{i}^{\dagger}(n) \\
= & 1+a^{2} F_{i j}+\frac{a^{3}}{2}\left(D_{i}-D_{j}\right) F_{i j}+\frac{a^{4}}{6}\left(D_{i}^{2}+D_{j}^{2}-\frac{3}{2} D_{j} D_{i}\right) F_{i j} \\
& +P_{i j}+\frac{a^{6}}{6} F_{i j}^{3}+O\left(a^{7}\right) .
\end{aligned}
$$

One can prove that the expansion of the charge conjugacy parity of above operator $U^{(i)}$ is equivalent to replace $F_{i j}$ by $-F_{i j}$ in Eq. (17) - (20).

Now we can expand arbitrary Wilson loop on lattice using above equations. It is shown in the following section. 


\section{The Expansion of Arbitrary Wilson Loop on Lattice}

We now give a general properties of the perturbative expansion of Wilson loops on lattice before we perform the expansion.

a Because differential operator can be presented by combination of difference operators with various ranks, the final contributions of full differential terms are zero due to the periodic boundary condition and we shall ignore such full differential terms in the expansion. In this reason, one can also expand Wilson loops on any lattice site with the same result.

In this reason, one can ignore full (covariant) terms with rank large than or equal to $a^{5}$ in section 2 and 3 in our case.

b On lattice an arbitrary Wilson loop can be expanded as

$$
O(n)=\sum_{k=0}^{\infty} a^{k} O_{k}(n),
$$

where $O_{k}(n)$, with mass-dimension $k$, is gauge-invariant polynomial of $A_{\mu}$ and $F_{\mu \nu}$ and their derivatives. In above equation even $k$ corresponds to parity $P=+$ while odd $k$ correspond to parity $P=-$.

c As we know, the $C$ parity of the real part of the operator is positive and that of the imaginary part is negative. Since the transformation of covariant differential $D_{i}\left(D_{i} \cdot=\partial_{i} \cdot-i\left[A_{i}, \cdot\right]\right)$ can be regard to be $1^{-+}$under $S O(3)^{P C}$ group in this case, we can regard $D_{i}$ as a real operator in the expansion.

One can simplify the expansion using three above properties. For instance, from above we know that $P_{i j}$ corresponds to $P C=++$ and it is invariant under translation on lattice if we only expand operators up to precision $O\left(a^{6}\right)$.

Utilizing the perturbative expansion of $U^{(i)}(i=1,2,3,4)$ in (17) - (20) one can expand an arbitrary Wilson loop on lattice. we exemplify it here.

The expansion of operators with $P C=++$ is the most complicated and we should only focus on it. So, when we say an gauge-invariant operator $L$ here we actually means $\frac{1}{4}(1+\widehat{C}+\widehat{P}+\widehat{C} \widehat{P}) L$, where $\widehat{P}$ and $\widehat{C}$ are parity operator and charge conjugacy parity operator which acts on operator $L$.

Let us observe the operator described in Fig. 4 and only care terms with $P C=++$, so that we may only keep real terms with even $k$ in Eq. (21).

$$
L=\operatorname{Tr}(1 \cdot 2 \cdot 3 \cdot \overline{4} \cdot \overline{5} \cdot \overline{6} \cdot \overline{7} \cdot 8),
$$

where, for simplification, we denote, for instance, the link variable $U(1)$ by 1 and $U^{\dagger}(1)$ by $\overline{1}$, etc. 
We rewrite $L$ as

$$
\begin{aligned}
L & =\operatorname{Tr}[1 \cdot 2 \cdot 3 \cdot \overline{4} \cdot \overline{9} \cdot \overline{1} \cdot 1 \cdot 9 \cdot \overline{5} \cdot \overline{10} \cdot 10 \cdot \overline{6} \cdot \overline{7} \cdot 8] \\
& =\operatorname{Tr}\left[p l_{1} \cdot p l_{2} \cdot p l 3\right],
\end{aligned}
$$

where $p l_{1}=1 \cdot 2 \cdot 3 \cdot \overline{4} \cdot \overline{9} \cdot \overline{1}, p l_{2}=1 \cdot 9 \cdot \overline{5} \cdot \overline{10}$ and $p l_{3}=10 \cdot \overline{6} \cdot \overline{7} \cdot 8$. Each of the $p l$ is gauge covariant respect to point $O$ under local gauge transformation.

Using equations in section 3 , we get

$$
\begin{aligned}
p l_{1}= & 1+a^{2} F_{i k}+\frac{a^{3}}{2}\left(D_{i}+D_{k}+2 D_{j}\right) F_{i k} \\
& +\frac{a^{4}}{6}\left(D_{i}^{2}+D_{k}^{2}+3 D_{j}^{2}+\frac{3}{2} D_{i k}+3 D_{j i}+3 D_{j k}\right) F_{i k}+P_{i k}+O\left(a^{7}\right),
\end{aligned}
$$

where $D_{i k}=D_{i} D_{k}$, etc.

$$
p l_{2}=1+a^{2} F_{j k}+\frac{a^{3}}{2}\left(D_{j}+D_{k}\right) F_{j k}+\frac{a^{4}}{6}\left(D_{j}^{2}+D_{k}^{2}+\frac{3}{2} D_{j k}\right) F_{j k}+O\left(a^{7}\right) .
$$

and

$$
p l_{3}=1+a^{2} F_{i k}-\frac{a^{3}}{2}\left(D_{i}-D_{k}\right) F_{i k}+\frac{a^{4}}{6}\left(D_{i}^{2}+D_{k}^{2}-\frac{3}{2} D_{i k}\right) F_{i k}+O\left(a^{7}\right) .
$$

So, up to $O\left(a^{6}\right)$, we have:

$$
\begin{aligned}
L= & 1+2 a^{4} \operatorname{Tr}\left(F_{i k} F_{i k}\right)+\frac{a^{4}}{2} \operatorname{Tr}\left(F_{j k} F_{j k}\right)+2 a^{4} \operatorname{Tr}\left(F_{i k} F_{j k}\right) \\
& +\frac{a^{6}}{6} \operatorname{Tr}\left[F_{i k}\left(4 D_{i}^{2}+D_{k}^{2}+3 D_{j}^{2}+6 D_{i j}\right) F_{i k}\right]+\frac{a^{6}}{24} \operatorname{Tr}\left[F_{j k}\left(D_{j}^{2}+D_{k}^{2}\right) F_{j k}\right] \\
& +\frac{a^{6}}{6} \operatorname{Tr}\left[F_{i k}\left(2 D_{i}^{2}+D_{j}^{2}+D_{k}^{2}+3 D_{i j}\right) F_{j k}\right]+O\left(a^{7}\right) .
\end{aligned}
$$

Here we have ignored terms with $P C \neq++$ and full covariant differential terms. Of course, we can do as similar for terms with $P C \neq++$ in the expansion.

\section{Conclusion}

We show an approach in the paper for the expansion of the Wilson loops on lattice. The approach presented here can determine the perturbative expansion of Wilson loop on lattice unambiguously according to $P$ and $C$ and is suitable for any Wilson loop on lattice despite how tanglesome the Wilson loop is.

It is also an appropriate method to discuss the improved action or improved cooling on lattice, etc. In fact, in some of the former papers which discuss improved action, there maybe exist some terms in higher precision which are not invariant under the rotation in $S O(4)$ group as the continuum theory requires, and therefore should bring up some artifact on lattice. Using this approach may help us to eliminate those unwanted terms if we discuss the improved action or other interesting problem up to higher precision. 


\section{References}

[1] K. Symanzik, Nucl. Phys. B226(1983) 187.

[2] M. G. Perez et al, Nucl. Phys. B413(1994) 535.

[3] F. Farchioni and A. Papa, Phys.Rev. D58(1998) 054502.

[4] D. Q. Liu et al, hep-lat/0103018.

[5] M. Luscher and P. Weisz, Commun. Math. Phys. 97(1985) 59.

[6] B. Berg and A. Billoire, Nucl. Phys. B221(1983) 109.

[7] D. Q. Liu et al, hep-lat/0011087.

[8] O. Nachtmann: "High Energy Collision and Nonperturbative QCD" in the lecture notes at the workshop "Topics in Field Theories" held on Oct. 1993, Kloster Banz, Germany.

[9] I. Are'feva, Teoreticheskaya i Matematicheskaya Fizica, 43(1980) 111(translation p. 353).

[10] Mandula, Zweig, Govaerts, Nucl. Phys. B228(1983) 109. 


\section{Figure captions}

- Figure 1 The operator $U_{R}\left(Z, O ; C_{X}\right) W(Z) U_{R}\left(O, Z ; \bar{C}_{X}\right)$, where $U_{R}$ is the connector described in the paper.

- Figure 2 The operator $U_{R}\left(Z, O ; C_{Y}+C_{X}\right) W(Z) U_{R}\left(O, Z ; \bar{C}_{X}+\bar{C}_{Y}\right)$, where the connector $U_{R}\left(Z, O ; C_{Y}+C_{X}\right)=U_{R}\left(Y, O ; C_{Y}\right) \cdot U_{R}\left(Z, Y ; C_{X}\right)$.

- Figure 3 The operators $U^{(i)}$ which are from point $O$ and back to point $O$. They are of the same shape with the different starting and ending point.

- Figure 4 The operator $L=\operatorname{Tr}(1 \cdot 2 \cdot 3 \cdot \overline{4} \cdot \overline{5} \cdot \overline{6} \cdot \overline{7} \cdot 8)$. Here we denote the link variable $U(i)(i=1,2, \cdots, 10)$ by $i$ and $U^{\dagger}(i)$ by $\bar{i}$. 


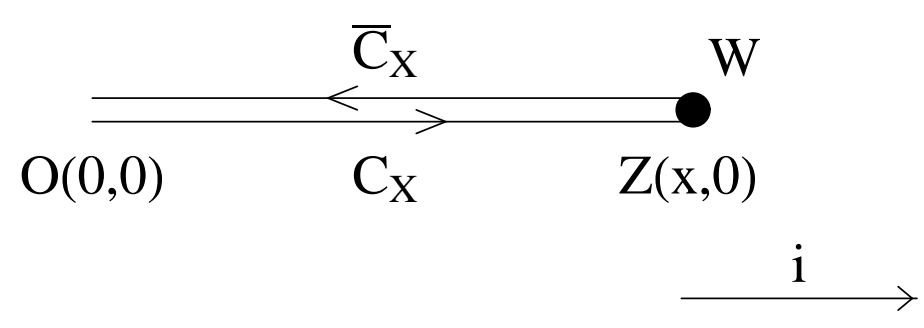

Figure 1 


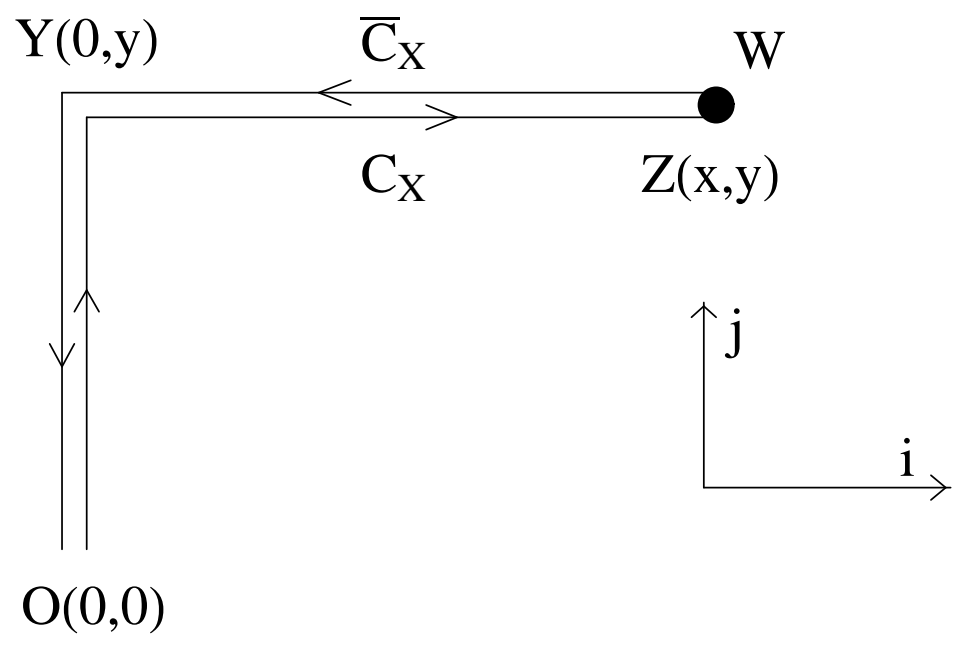

Figure 2 


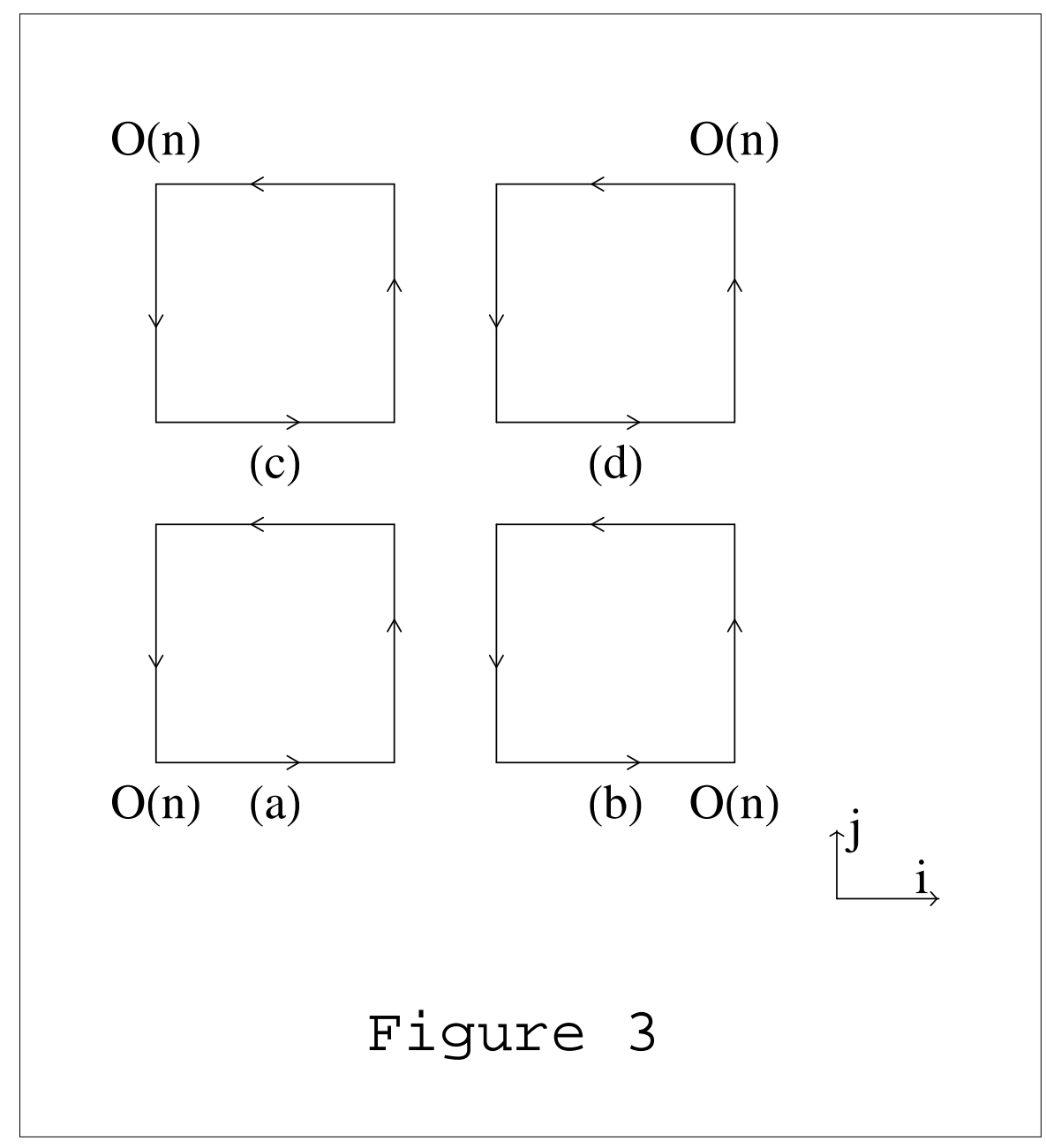




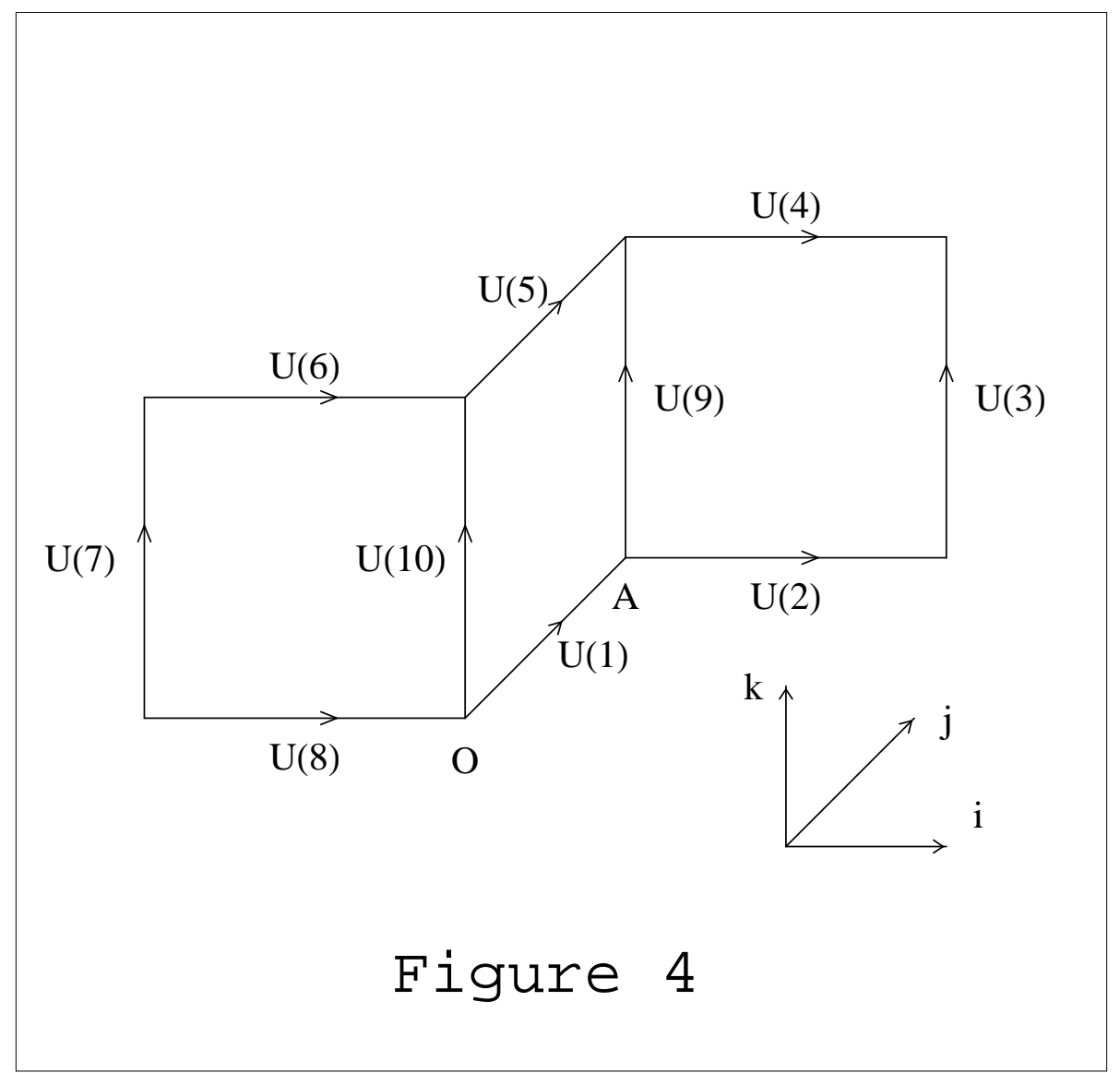

